\title{
Semi-infinite metal: perturbative treatment based on semi-infinite jellium
}

\author{
P.P.Kostrobij, B.M.Markovych \\ Lviv Polytechnic National University, 12, S. Bandera Str., Lviv 79013, Ukraine
}

Received February 27, 2008

\begin{abstract}
Energy of electronic subsystem of semi-infinite metal is presented in the form of an expansion in powers of pseudo-potential. It is shown that generally electron many-particle density matrices are necessary for the energy calculation, whereas in case of a local pseudo-potential only diagonal elements (electron distribution functions) are necessary. In a specific case of a local pseudo-potential within the first order of perturbation theory, our results for energy coincide with those widely applicable in the density functional theory.
\end{abstract}

Key words: partition function, pseudo-potential, many-particle density matrix

PACS: $71.45 . G m, 71.10 .-w, 73.20 .-r$

\section{Introduction}

Theoretical studies of equilibrium properties of a metal surface turn out to be very difficult, because an electronic subsystem of a bounded metal is spatially very nonuniform. This greatly complicates the consecutive account of many-particle effects in an electronic subsystem. The greatest successes in studying the electronic properties of a metal surface have been attained within density functional theory (DFT) [1]. However, the study of properties of a metal surface which are caused by discreteness of the ionic subsystem is quite problematic, since there is no technique for constructing necessary energy functionals.

The first attempt in this direction was made by Lang and Kohn [2]. At first they used a time jellium model for a metal surface with the correction that takes into consideration discreteness of the ionic subsystem. This correction was simple enough: self-consistent density was still that of jellium while the perturbation (the difference between the lattice potential and that of the uniform positive background) was averaged over the surface plane. Thus, they managed to consider the ion cores via the first order perturbation theory and via the classical cleavage energy. In subsequent works [3-6] surface energy calculations have been fulfilled taking into account the discreteness of the ionic subsystem in the first order of the perturbation and with the use of variational methods. These calculations showed that in case of metals with ionic charge $\mathcal{Z}=1,2$ the surface energy is in good agreement with experimental data. Whereas for charges $\mathcal{Z}>2$ this model is not adequate. The firstorder perturbative results based on jellium for the surface energy of slabs of simple metals, using various local pseudopotentials (Ashcroft, Heine-Abarenkov and evanescent core) were examined in work [7].

Later, discreteness of the ionic subsystem was taken into account by Rose and Dobson $[8,9]$ via the second-order perturbation theory, which includes the linear response function of semi-infinite jellium. They calculated the second-order surface energy terms. At the same time they used the linear response of bulk jellium, in a kind of local approximation. Second-order perturbation theory using the linear response of a jellium slab in the random phase approximation has been worked out by Barnett and coworkes [10] as well as by Eguiluz [11]. These calculations show a noticeable effect of the second-order term in the face-dependent surface energies. A systematic method for constructing a perturbation theory for energy of an electronic subsystem in the field of static ions in non-transitive metals having a surface was offered by Kaim [12]. 
The structureless pseudo-potential model which had been applied to the calculations of a metal surface was developed in works [13-15]. This model is nothing else but a well-known zero model of metal [16-19]. In this model the energy of nonuniform metal consists of the energy of electronic gas in a positive jellium, the Madelung energy of pointwise ions in homogeneous electron density, the energy caused by non-Coulomb character of an electron-ion interaction at a zero transfer momentum and the value of the first-order pseudo-potential correction averaged by volume of semi-infinite metal. This average is included in the self-consistent procedure of Lang and Kohn [2]. The derived values of a surface energy of simple metals are close to those in the paper [2].

Thus, a certain progress in the theory of metal surface has been reached, but mainly for the surfaces of simple metals that can be adequately described by local pseudo-potentials. Therefore, in the presented paper, systematic perturbation theory for bounded metals, described by nonlocal pseudo-potentials, is developed. The semi-infinite jellium [20-23] is used as a reference system and the perturbation theory with respect to the "difference potential" ${ }^{1}$ is constructed. In a specific case of a local pseudopotential, this perturbation theory coincides with the results by Kaim [12].

In section 2, the model of semi-infinite metal is described, the definition of surface potential is entered, a Hamiltonian of this system is written in the second-quantization form. In section 3, the partition function is presented in the form of an expansion in powers of pseudopotential. Section 4 presents a specific case of local pseudo-potential and a comparison with the results of other papers. Conclusions are presented in section 5. Appendix presents a useful proof of the statement.

\section{Model}

We consider a semi-infinite metal with ions having charges $\mathcal{Z} e$ and Cartesian coordinates $\mathbf{R}_{j}$ $\left(-\infty<X_{j}, Y_{j}<+\infty, Z_{j} \leqslant Z_{0}, Z_{0}=\right.$ const, $z=Z_{0}$ is the division plane (surface)), $j=1, \ldots, N_{\text {ion }}$. Electrons of a semi-infinite metal have coordinates $\mathbf{r}_{i}, i=1, \ldots, N$. A Hamiltonian of this model has the following form

$$
H=-\frac{\hbar^{2}}{2 m} \sum_{i=1}^{N} \Delta_{i}+\frac{1}{2} \sum_{i \neq j=1}^{N} \frac{e^{2}}{\left|\mathbf{r}_{i}-\mathbf{r}_{j}\right|}+\sum_{j=1}^{N_{\text {ion }}} \frac{\mathbf{P}_{j}^{2}}{2 M}+\frac{1}{2} \sum_{i \neq j=1}^{N_{\text {ion }}} \frac{(\mathcal{Z} e)^{2}}{\left|\mathbf{R}_{i}-\mathbf{R}_{j}\right|}+\sum_{i=1}^{N} \sum_{j=1}^{N_{\text {ion }}} w\left(\mathbf{r}_{i}, \mathbf{R}_{j}\right)
$$

where the first summand is the kinetic energy of electrons, the second summand represents the potential energy of the interelectron interaction, the third summand is the kinetic energy of ions ( $\mathbf{P}$ is the operator of ion momentum), the fourth summand represents the potential energy of the ion-ion interaction and the last one represents the energy of electron-ion interaction. We assume that the system is electroneutral, that is

$$
\mathcal{Z} N_{\text {ion }}=N \text {. }
$$

We shall present the potential of the electron-ionic interaction as

$$
w\left(\mathbf{r}_{i}, \mathbf{R}_{j}\right)=w\left(\left|\mathbf{r}_{i}-\mathbf{R}_{j}\right|\right)+\Delta w\left(\mathbf{r}_{i}, \mathbf{R}_{j}\right),
$$

where $w\left(\left|\mathbf{r}_{i}-\mathbf{R}_{j}\right|\right)$ is a periodic potential of the electron-ion interaction in the case of infinite metal (pseudo-potential), $\Delta w\left(\mathbf{r}_{i}, \mathbf{R}_{j}\right)$ is a deviation of the electron-ionic potential of the semiinfinite metal from the periodic one.

From the Hamiltonian (1) we extract a Hamiltonian of the semi-infinite jellium model $H_{\text {jell }}$ which was explored in papers [20-22]. We shall use the model of semi-infinite metal as the reference system for studying thermodynamic and structural properties of semi-infinite metal. Finally we get

$$
H=H_{\mathrm{jell}}+\delta H_{\mathrm{ii}}+\sum_{i=1}^{N} \sum_{j=1}^{N_{\mathrm{ion}}} \delta w\left(\left|\mathbf{r}_{i}-\mathbf{R}_{j}\right|\right)
$$

\footnotetext{
${ }^{1}$ That is the difference between the pseudo-potential of ions and the electrostatic potential of the semi-infinite jellium positive background.
} 
where

$$
\begin{gathered}
\delta H_{\text {ii }}=\sum_{j=1}^{N_{\text {ion }}} \frac{\mathbf{P}_{j}^{2}}{2 M}+\frac{1}{2} \sum_{i \neq j=1}^{N_{\text {ion }}} \frac{(\mathcal{Z} e)^{2}}{\left|\mathbf{R}_{i}-\mathbf{R}_{j}\right|}-\frac{1}{2} \int \mathrm{d} \mathbf{R} \int \mathrm{d} \mathbf{R}^{\prime} \frac{\rho_{\text {jell }}(\mathbf{R}) \rho_{\text {jell }}\left(\mathbf{R}^{\prime}\right)}{\left|\mathbf{R}-\mathbf{R}^{\prime}\right|} \\
H_{\text {jell }}=H_{\text {jell }}^{\text {unif }}+\sum_{i=1}^{N} V\left(\mathbf{r}_{i}\right) \\
V\left(\mathbf{r}_{i}\right)=V_{\text {jell }}\left(\mathbf{r}_{i}\right)+V_{\text {ion }}\left(\mathbf{r}_{i}\right)
\end{gathered}
$$

is the surface potential acting on electrons.

$$
\delta w\left(\left|\mathbf{r}_{i}-\mathbf{R}_{j}\right|\right)=w\left(\left|\mathbf{r}_{i}-\mathbf{R}_{j}\right|\right)+\frac{1}{N_{\text {ion }}} \int \mathrm{d} \mathbf{R} \frac{e \rho_{\text {jell }}(\mathbf{R})}{\left|\mathbf{r}_{i}-\mathbf{R}\right|}
$$

is the "difference potential" (see footnote on page 642),

$$
H_{\text {jell }}^{\text {unif }}=-\frac{\hbar^{2}}{2 m} \sum_{i=1}^{N} \Delta_{i}+\frac{1}{2} \sum_{i \neq j=1}^{N} \frac{e^{2}}{\left|\mathbf{r}_{i}-\mathbf{r}_{j}\right|}-\frac{e^{2} N}{V} \sum_{i=1}^{N} \int \mathrm{d} \mathbf{R} \frac{1}{\left|\mathbf{r}_{i}-\mathbf{R}\right|}+\frac{(e N)^{2}}{2 V^{2}} \int \mathrm{d} \mathbf{R} \int \mathrm{d} \mathbf{R}^{\prime} \frac{1}{\left|\mathbf{R}-\mathbf{R}^{\prime}\right|}
$$

is the Hamiltonian of homogeneous jellium. $V=S L$ is the volume of the system, $S$ is the surface area of semi-infinite metal, $L$ determines the area of the change of the electron coordinate normal to the surface: $z \in(-L / 2,+L / 2), S \rightarrow \infty, L \rightarrow \infty$.

$$
V_{\text {jell }}\left(\mathbf{r}_{i}\right)=e \int \mathrm{d} \mathbf{R} \frac{e N / V-\rho_{\text {jell }}(\mathbf{R})}{\left|\mathbf{r}_{i}-\mathbf{R}\right|}+\frac{1}{2 N} \int \mathrm{d} \mathbf{R} \int \mathrm{d} \mathbf{R}^{\prime} \frac{\rho_{\text {jell }}(\mathbf{R}) \rho_{\text {jell }}\left(\mathbf{R}^{\prime}\right)-(e N / V)^{2}}{\left|\mathbf{R}-\mathbf{R}^{\prime}\right|}
$$

is the part of the surface potential formed by semi-infinite jellium.

$$
V_{\text {ion }}\left(\mathbf{r}_{i}\right)=\sum_{j=1}^{N_{\text {ion }}} \Delta w\left(\mathbf{r}_{i}, \mathbf{R}_{j}\right)
$$

is the part of the surface potential created by deviation $\Delta w\left(\mathbf{r}_{i}, \mathbf{R}_{j}\right)$ of the true electron-ionic potential of semi-infinite metal $w\left(\mathbf{r}_{i}, \mathbf{R}_{j}\right)$ from the space-periodical electron-ionic potential $w\left(\mid \mathbf{r}_{i}-\right.$ $\left.\mathbf{R}_{j} \mid\right)$.

$$
\rho_{\text {jell }}(\mathbf{R}) \equiv \rho_{\text {jell }}(Z)=\rho_{0} \theta(-d-Z), \quad \rho_{0}=\frac{e N}{V / 2}
$$

is a distribution of the ionic density in semi-infinite jellium, parameter $d$ is defined by the electroneutrality condition.

In what follows we assume that the electron-ionic potential $w\left(\left|\mathbf{r}_{i}-\mathbf{R}_{j}\right|\right)$ is modelled by a nonlocal model pseudo-potential [24]

$$
w\left(\left|\mathbf{r}_{i}-\mathbf{R}_{j}\right|\right)=-\frac{\mathcal{Z} e^{2}}{\left|\mathbf{r}_{i}-\mathbf{R}_{j}\right|}+\sum_{l^{\prime}=0}^{l} f_{l^{\prime}}\left(\left|\mathbf{r}_{i}-\mathbf{R}_{j}\right|\right) \mathrm{P}_{l^{\prime}},
$$

where

$$
\mathrm{P}_{l}=\sum_{m=-l}^{l}\left|\mathrm{Y}_{l, m}\right\rangle\left\langle\mathrm{Y}_{l, m}\left|\equiv \sum_{m=-l}^{l}\right| l, m\right\rangle\langle l, m|
$$

is the projection operator, and

$$
\sum_{l} \mathrm{P}_{l}=1
$$

$l$ and $m$ are the electronic orbital and magnetic quantum numbers, respectively. The concrete expression for function $f_{l}\left(\left|\mathbf{r}_{i}-\mathbf{R}_{j}\right|\right)$ depends on the selected model of pseudo-potential. 
For a further discussion it is convenient to rewrite the Hamiltonian (10) in the second quantization form. For this purpose, we introduce wave functions $\psi_{f}(\mathbf{r})$ of an electron being in the surface potential $V(\mathbf{r})$

$$
\left[-\frac{\hbar^{2}}{2 m} \Delta+V(\mathbf{r})\right] \psi_{f}(\mathbf{r})=E_{f} \psi_{f}(\mathbf{r})
$$

Then the field operators are introduced by definition

$$
\begin{aligned}
\Psi(\mathbf{r}) & =\sum_{f} \psi_{f}(\mathbf{r}) a_{f}, \\
\Psi^{\dagger}(\mathbf{r}) & =\sum_{f} \psi_{f}^{*}(\mathbf{r}) a_{f}^{\dagger},
\end{aligned}
$$

where $a_{f}$ and $a_{f}^{\dagger}$ are the annihilation and creation operators of electrons in the state $f$ with an energy $E_{f}$ respectively,

$$
\left\{a_{f}, a_{f^{\prime}}^{\dagger}\right\}=\delta_{f, f^{\prime}}
$$

Since it is very difficult to solve the equation (16), we assume that the surface potential $V(\mathbf{r})$ is a function of the electron coordinate normal to the surface

$$
V(\mathbf{r}) \equiv V(z)
$$

Then, the electron wave function is the product of plane wave and function depending only on coordinate $z$ :

$$
\psi_{f}(\mathbf{r})=\frac{1}{\sqrt{S}} \mathrm{e}^{\mathrm{i} \mathbf{p r} \|_{\alpha}} \varphi_{\alpha}(z), \quad \mathbf{r}=\left(\mathbf{r}_{\|}, z\right), f=(\mathbf{p}, \alpha)
$$

where function $\varphi_{\alpha}(z)$ is a solution of the following equation

$$
\left[-\frac{\hbar^{2}}{2 m} \frac{\mathrm{d}^{2}}{\mathrm{~d} z^{2}}+V(z)\right] \varphi_{\alpha}(z)=\varepsilon_{\alpha} \varphi_{\alpha}(z)
$$

Then, $E_{f} \equiv E_{\alpha}(\mathbf{p})=\frac{\hbar^{2} \mathbf{p}^{2}}{2 m}+\varepsilon_{\alpha}$ is the energy of electron in a state $(\mathbf{p}, \alpha), \hbar \mathbf{p}$ is a two-dimensional momentum of electron in the plane parallel to the surface.

Then, the Hamiltonian (4) can be written as

$$
H=H_{\text {jell }}+\delta H_{\mathrm{ii}}+\frac{N_{\mathrm{ion}}}{S L} \sum_{\mathbf{q}} \sum_{k} \mathrm{~S}_{k}(\mathbf{q}) \sum_{l} \delta w_{k}^{l}(\mathbf{q}) \tilde{\rho}_{k}^{l}(\mathbf{q}),
$$

where

$$
\mathrm{S}_{k}(\mathbf{q})=\frac{1}{N_{\text {ion }}} \sum_{j=1}^{N_{\text {ion }}} \mathrm{e}^{-\mathrm{iq} \mathbf{R}_{|| j}-\mathrm{i} k Z_{j}}
$$

is a geometrical structure factor of the ionic subsystem of semi-infinite metal,

$$
\delta w_{k}^{l}(\mathbf{q})=-\mathcal{Z} \nu_{k}(\mathbf{q})\left(1-\delta_{\mathbf{q}, 0}\right)+f_{k}^{l}(\mathbf{q})
$$

$\nu_{k}(\mathbf{q})=4 \pi e^{2} /\left(q^{2}+k^{2}\right)$ and $f_{k}^{l}(\mathbf{q})$ are three-dimensional Fourier-image of Coulomb potential and nonlocal part of the pseudo-potential (13):

$$
\begin{gathered}
\frac{e^{2}}{\left|\mathbf{r}_{i}-\mathbf{r}_{j}\right|}=\frac{1}{S L} \sum_{\mathbf{q}, k} \nu_{k}(\mathbf{q}) \mathrm{e}^{\mathrm{i} \mathbf{q}\left(\mathbf{r}_{\| i}-\mathbf{r}_{\mid j}\right)+\mathrm{i} k\left(z_{i}-z_{j}\right)}, \\
f_{l}\left(\left|\mathbf{r}_{i}-\mathbf{R}_{j}\right|\right)=\frac{1}{S L} \sum_{\mathbf{q}, k} f_{k}^{l}(\mathbf{q}) \mathrm{e}^{\mathrm{i} \mathbf{q}\left(\mathbf{r}_{|| i}-\mathbf{R}_{|| j}\right)+\mathrm{i} k\left(z_{i}-Z_{j}\right)}, \quad \mathbf{R}_{\| j}=\left(X_{j}, Y_{j}\right), \\
\tilde{\rho}_{k}^{l}(\mathbf{q})=\sum_{m} \sum_{\mathbf{p}_{1}, \alpha_{1}} \sum_{\mathbf{p}_{2}, \alpha_{2}}\left\langle\mathbf{p}_{1}, \alpha_{1}\left|\mathrm{e}^{\mathrm{i} \mathbf{q} \mathbf{r}_{\| \mid}+\mathrm{i} k z}\right| l, m\right\rangle\left\langle l, m \mid \mathbf{p}_{2}, \alpha_{2}\right\rangle a_{\alpha_{1}}^{\dagger}\left(\mathbf{p}_{1}\right) a_{\alpha_{2}}\left(\mathbf{p}_{2}\right),
\end{gathered}
$$




$$
\begin{aligned}
\left\langle\mathbf{p}_{1}, \alpha_{1}|\ldots| l, m\right\rangle & =\frac{1}{\sqrt{S}} \int \mathrm{d} \mathbf{r}_{\|} \int \mathrm{d} z \mathrm{e}^{-\mathrm{i} \mathbf{p}_{1} \mathbf{r}_{\|}} \varphi_{\alpha_{1}}^{*}(z) \ldots \mathrm{Y}_{l, m}(\theta, \phi), \\
\left\langle l, m \mid \mathbf{p}_{2}, \alpha_{2}\right\rangle & =\frac{1}{\sqrt{S}} \int \mathrm{d} \mathbf{r}_{\|} \int \mathrm{d} z \mathrm{Y}_{l, m}^{*}(\theta, \phi) \mathrm{e}^{\mathrm{i} \mathbf{p}_{2} \mathbf{r}_{\|}} \varphi_{\alpha_{2}}(z) .
\end{aligned}
$$

Let us note that in the case of a local pseudo-potential, the Hamiltonian (18) acquires the following form:

$$
H=H_{\text {jell }}+\delta H_{\mathrm{ii}}+\frac{N_{\text {ion }}}{S L} \sum_{\mathbf{q}} \sum_{k} \mathrm{~S}_{k}(\mathbf{q}) \delta w_{k}(\mathbf{q}) \rho_{k}(\mathbf{q})
$$

where

$$
\begin{aligned}
\delta w_{k}(\mathbf{q}) & =-\mathcal{Z} \nu_{k}(\mathbf{q})\left(1-\delta_{\mathbf{q}, 0}\right)+f_{k}(\mathbf{q}) \\
\rho_{k}(\mathbf{q}) \equiv \sum_{l} \tilde{\rho}_{k}^{l}(\mathbf{q}) & =\sum_{\mathbf{p}, \alpha_{1}, \alpha_{2}}\left\langle\alpha_{1}\left|\mathrm{e}^{\mathrm{i} k z}\right| \alpha_{2}\right\rangle a_{\alpha_{1}}^{\dagger}(\mathbf{p}) a_{\alpha_{2}}(\mathbf{p}-\mathbf{q}) \\
\left\langle\alpha_{1}|\ldots| \alpha_{2}\right\rangle & =\int \mathrm{d} z \varphi_{\alpha_{1}}^{*}(z) \ldots \varphi_{\alpha_{2}}(z)
\end{aligned}
$$

Such representation of the Hamiltonian (see (18) or (21)) is convenient for calculation of the partition function, which indicates thermodynamic characteristics of the system.

Let us note that since the electron has two possible orientations of a spin, a result of summation on $\mathbf{p}$ in the formula (20) (or in (22)) should be doubled.

\section{Partition function}

We consider the partition function of the semi-infinite metal

$$
\Xi=\operatorname{Sp~} \mathrm{e}^{-\beta(H-\mu \mathcal{N})}
$$

where $\mu$ is a chemical potential of the electronic subsystem, $\mathcal{N}$ is an electron number operator. Taking into account (18), in adiabatic approximation we get

$$
\Xi=\mathrm{e}^{-\beta \delta H_{\mathrm{ii}}} \operatorname{Sp~} \mathrm{e}^{-\beta\left(\mathcal{H}_{\mathrm{jell}}-\delta V_{\mathrm{ei}}\right)},
$$

where

$$
\begin{gathered}
\mathcal{H}_{\text {jell }}=H_{\text {jell }}-\mu \mathcal{N} \\
\delta V_{\text {ei }}=\frac{N_{\text {ion }}}{S L} \sum_{\mathbf{q}} \sum_{k} \mathrm{~S}_{k}(\mathbf{q}) \sum_{l} \delta w_{k}^{l}(\mathbf{q}) \tilde{\rho}_{k}^{l}(\mathbf{q}) .
\end{gathered}
$$

In the interaction representation, the partition function is presented as

$$
\Xi=\mathrm{e}^{-\beta \delta H_{\mathrm{ii}}} \Xi_{\text {jell }}\langle\mathcal{S}(\beta)\rangle_{\text {jell }},
$$

where

$$
\Xi_{\text {jell }}=\operatorname{Sp~} \mathrm{e}^{-\beta \mathcal{H}_{\text {jell }}}
$$

is the partition function of semi-infinite jellium [20] ( $\mathrm{T}$ is the time-ordering operator),

$$
\begin{gathered}
\langle\ldots\rangle_{\text {jell }}=\frac{1}{\Xi_{\text {jell }}} \operatorname{Sp}\left(\mathrm{e}^{-\beta \mathcal{H}_{\text {jell }}} \ldots\right) \\
\mathcal{S}(\beta)=\mathrm{T} \exp \left[-\frac{N_{\text {ion }}}{S L} \int_{0}^{\beta} \mathrm{d} \beta^{\prime} \sum_{\mathbf{q}} \sum_{k} \mathrm{~S}_{k}(\mathbf{q}) \sum_{l} \delta w_{k}^{l}(\mathbf{q}) \tilde{\rho}_{k}^{l}\left(\mathbf{q} \mid \beta^{\prime}\right)\right] \\
\tilde{\rho}_{k}^{l}\left(\mathbf{q} \mid \beta^{\prime}\right)=\mathrm{e}^{\beta^{\prime} \mathcal{H}_{\text {jell }}} \tilde{\rho}_{k}^{l}(\mathbf{q}) \mathrm{e}^{-\beta^{\prime} \mathcal{H}_{\text {jell }}} .
\end{gathered}
$$


Transferring from the temperature representation to the frequency representation, according to the rule:

$$
\begin{aligned}
& \tilde{\rho}_{k}^{l}(\mathbf{q} \mid \nu)=\frac{1}{\beta} \int_{0}^{\beta} \mathrm{d} \beta^{\prime} \mathrm{e}^{\mathrm{i} \nu \beta^{\prime}} \tilde{\rho}_{k}^{l}\left(\mathbf{q} \mid \beta^{\prime}\right), \\
& \tilde{\rho}_{k}^{l}\left(\mathbf{q} \mid \beta^{\prime}\right)=\sum_{\nu} \mathrm{e}^{-\mathrm{i} \nu \beta^{\prime}} \tilde{\rho}_{k}^{l}(\mathbf{q} \mid \nu),
\end{aligned}
$$

where $\nu$ is the Bose frequency, we get

$$
\mathcal{S}(\beta)=\mathrm{T} \exp \left[-\frac{\beta N_{\text {ion }}}{S L} \sum_{\mathbf{q}} \sum_{k} \mathrm{~S}_{k}(\mathbf{q}) \sum_{l} \delta w_{k}^{l}(\mathbf{q}) \tilde{\rho}_{k}^{l}(\mathbf{q} \mid \nu=0)\right]
$$

and

$$
\begin{aligned}
\langle\mathcal{S}(\beta)\rangle_{\text {jell }}= & \exp \left[\sum_{n=1}^{\infty}\left(\frac{\beta N_{\text {ion }}}{S L}\right)^{n} \frac{\mathrm{i}^{n}}{n !} \sum_{\mathbf{q}_{1}, \ldots, \mathbf{q}_{n}} \sum_{k_{1}, \ldots, k_{n}} \mathrm{~S}_{k_{1}}\left(\mathbf{q}_{1}\right) \ldots \mathrm{S}_{k_{n}}\left(\mathbf{q}_{n}\right)\right. \\
& \left.\times \sum_{l_{1}, \ldots, l_{n}} \delta w_{k_{1}}^{l_{1}}\left(\mathbf{q}_{1}\right) \ldots \delta w_{k_{n}}^{l_{n}}\left(\mathbf{q}_{n}\right) \mathfrak{M}_{k_{1}, \ldots, k_{n}}^{l_{1}, \ldots, l_{n}}\left(\mathbf{q}_{1}, \ldots, \mathbf{q}_{n} \mid \nu=0\right)\right]
\end{aligned}
$$

where

$$
\mathfrak{M}_{k_{1}, \ldots, k_{n}}^{l_{1}, \ldots, l_{n}}\left(\mathbf{q}_{1}, \ldots, \mathbf{q}_{n} \mid \nu=0\right)=\mathrm{i}^{n}\left\langle\mathrm{~T} \tilde{\rho}_{k_{1}}^{l_{1}}\left(\mathbf{q}_{1} \mid \nu=0\right) \ldots \tilde{\rho}_{k_{n}}^{l_{n}}\left(\mathbf{q}_{n} \mid \nu=0\right)\right\rangle_{\text {jell,c }}
$$

is the $n$th order irreducible correlation function of electrons.

Since the average value of the quantities $\tilde{\rho}_{k_{n}}^{l_{n}}\left(\mathbf{q}_{n} \mid \nu=0\right)$ is equal to the average value of the quantities $\tilde{\rho}_{k_{n}}^{l_{n}}\left(\mathbf{q}_{n}\right)$ (see Appendix) it is possible to write

$$
\begin{aligned}
\langle\mathcal{S}(\beta)\rangle_{\text {jell }}= & \exp \left[\sum_{n=1}^{\infty}\left(\frac{\beta N_{\text {ion }}}{S L}\right)^{n} \frac{\mathrm{i}^{n}}{n !} \sum_{\mathbf{q}_{1}, \ldots, \mathbf{q}_{n}} \sum_{k_{1}, \ldots, k_{n}} \mathrm{~S}_{k_{1}}\left(\mathbf{q}_{1}\right) \ldots \mathrm{S}_{k_{n}}\left(\mathbf{q}_{n}\right)\right. \\
& \left.\times \sum_{l_{1}, \ldots, l_{n}} \delta w_{k_{1}}^{l_{1}}\left(\mathbf{q}_{1}\right) \ldots \delta w_{k_{n}}^{l_{n}}\left(\mathbf{q}_{n}\right) \mathfrak{M}_{k_{1}, \ldots, k_{n}}^{l_{1}, \ldots, l_{n}}\left(\mathbf{q}_{1}, \ldots, \mathbf{q}_{n}\right)\right]
\end{aligned}
$$

where

$$
\mathfrak{M}_{k_{1}, \ldots, k_{n}}^{l_{1}, \ldots, l_{n}}\left(\mathbf{q}_{1}, \ldots, \mathbf{q}_{n}\right)=\mathrm{i}^{n}\left\langle\tilde{\rho}_{k_{1}}^{l_{1}}\left(\mathbf{q}_{1}\right) \ldots \tilde{\rho}_{k_{n}}^{l_{n}}\left(\mathbf{q}_{n}\right)\right\rangle_{\text {jell,c }}
$$

Calculation of $\mathfrak{M}_{k_{1}, \ldots, k_{n}}^{l_{1}, \ldots, l_{n}}\left(\mathbf{q}_{1}, \ldots, \mathbf{q}_{n}\right)$ can be made according to the definition (see (28)) using a perturbation theory, but for the sake of comparison of our theories with others it is more convenient to present $\mathfrak{M}_{k_{1}, \ldots, k_{n}}^{l_{1}, \ldots, l_{n}}\left(\mathbf{q}_{1}, \ldots, \mathbf{q}_{n}\right)$ through many-particle density matrices. According to Bogoljubov $[25]$ between $\mathfrak{M}_{k_{1}, \ldots, k_{n}}^{l_{1}, \ldots, l_{n}}\left(\mathbf{q}_{1}, \ldots, \mathbf{q}_{n}\right)$ and $s$-particle density matrix

$$
\frac{N(N-1) \ldots(N-s+1)}{V^{s}} \mathfrak{F}_{s}\left(\mathbf{r}_{1}, \ldots, \mathbf{r}_{s} \mid \mathbf{r}_{1}^{\prime}, \ldots, \mathbf{r}_{s}^{\prime}\right)
$$

there exists the following relation:

$$
\begin{aligned}
\mathfrak{F}_{s}\left(\mathbf{r}_{1}, \ldots, \mathbf{r}_{s} \mid \mathbf{r}_{1}^{\prime}, \ldots, \mathbf{r}_{s}^{\prime}\right)= & \frac{V^{s}}{N(N-1) \ldots(N-s+1)} \sum_{\substack{f_{1}, \ldots, f_{s} \\
f_{1}^{\prime}, \ldots, f_{s}^{\prime}}} \psi_{f_{1}}^{*}\left(\mathbf{r}_{1}\right) \ldots \psi_{f_{s}}^{*}\left(\mathbf{r}_{s}\right) \\
& \times \psi_{f_{1}^{\prime}}\left(\mathbf{r}_{1}^{\prime}\right) \ldots \psi_{f_{s}^{\prime}}\left(\mathbf{r}_{s}^{\prime}\right)\left\langle a_{f_{1}}^{\dagger} \ldots a_{f_{s}}^{\dagger} a_{f_{s}^{\prime}} \ldots a_{f_{1}^{\prime}}\right\rangle_{\text {jell }} .
\end{aligned}
$$


The use of orthogonality of the wave functions (17),

$$
\int \mathrm{d} \mathbf{r} \psi_{f_{1}}^{*}(\mathbf{r}) \psi_{f_{2}}(\mathbf{r})=\delta_{f_{1}, f_{2}},
$$

permits to write the expression (35) in the form

$$
\begin{aligned}
\left\langle a_{f_{1}}^{\dagger} \ldots a_{f_{s}}^{\dagger} a_{f_{s}^{\prime}} \ldots a_{f_{1}^{\prime}}\right\rangle_{\text {jell }}= & \frac{N(N-1) \ldots(N-s+1)}{V^{s}} \int \mathrm{d} \mathbf{r}_{1} \ldots \int \mathrm{d} \mathbf{r}_{s} \int \mathrm{d} \mathbf{r}_{1}^{\prime} \ldots \int \mathrm{d} \mathbf{r}_{s}^{\prime} \\
& \times \psi_{f_{1}}^{*}\left(\mathbf{r}_{1}\right) \ldots \psi_{f_{s}}^{*}\left(\mathbf{r}_{s}\right) \mathfrak{F}_{s}\left(\mathbf{r}_{1}, \ldots, \mathbf{r}_{s} \mid \mathbf{r}_{1}^{\prime}, \ldots, \mathbf{r}_{s}^{\prime}\right) \psi_{f_{1}^{\prime}}\left(\mathbf{r}_{1}^{\prime}\right) \ldots \psi_{f_{s}^{\prime}}\left(\mathbf{r}_{s}^{\prime}\right)
\end{aligned}
$$

Let us consider the first order correlation functions

$$
\begin{aligned}
\mathfrak{M}_{k_{1}}^{l_{1}}\left(\mathbf{q}_{1}\right) & =\mathrm{i}\left\langle\tilde{\rho}_{k_{1}}^{\mathcal{1}_{1}}\left(\mathbf{q}_{1}\right)\right\rangle_{\text {jell }} \\
& =\mathrm{i} \sum_{m} \sum_{\mathbf{p}_{1}, \alpha_{1}} \sum_{\mathbf{p}_{2}, \alpha_{2}}\left\langle\mathbf{p}_{1}, \alpha_{1}\left|\mathrm{e}^{\mathrm{i} \mathbf{q}_{1} \mathbf{r}_{||}+\mathrm{i} k_{1} z}\right| l_{1}, m\right\rangle\left\langle l_{1}, m \mid \mathbf{p}_{2}, \alpha_{2}\right\rangle\left\langle a_{\alpha_{1}}^{\dagger}\left(\mathbf{p}_{1}\right) a_{\alpha_{2}}\left(\mathbf{p}_{2}\right)\right\rangle_{\text {jell }} \\
& =\mathrm{i} \frac{N}{V} \sum_{m} \int \mathrm{d} \mathbf{r}_{1} \int \mathrm{d} \mathbf{r}_{1}^{\prime} \mathrm{e}^{\mathrm{i} \mathbf{q}_{1} \mathbf{r}_{|| 1}+\mathrm{i} k_{1} z_{1}} Y_{l_{1}, m}\left(\theta_{1}, \phi_{1}\right) \mathfrak{F}_{1}\left(\mathbf{r}_{1} \mid \mathbf{r}_{1}^{\prime}\right) Y_{l_{1}, m}^{*}\left(\theta_{1}^{\prime}, \phi_{1}^{\prime}\right)
\end{aligned}
$$

and the second order irreducible correlation functions

$$
\begin{aligned}
\mathfrak{M}_{k_{1}, k_{2}}^{l_{1}, l_{2}}\left(\mathbf{q}_{1}, \mathbf{q}_{2}\right)= & \mathrm{i}^{2}\left\langle\tilde{\rho}_{k_{1}}^{\mathcal{l}_{1}}\left(\mathbf{q}_{1}\right) \tilde{\rho}_{k_{2}}^{l_{2}}\left(\mathbf{q}_{2}\right)\right\rangle_{\mathrm{jell}, \mathrm{c}}=\mathrm{i}^{2}\left\langle\tilde{\rho}_{k_{1}}^{l_{1}}\left(\mathbf{q}_{1}\right) \tilde{\rho}_{k_{2}}^{l_{2}}\left(\mathbf{q}_{2}\right)\right\rangle_{\mathrm{jell}}-\mathrm{i}\left\langle\tilde{\rho}_{k_{1}}^{l_{1}}\left(\mathbf{q}_{1}\right)\right\rangle_{\mathrm{jell}} \mathrm{i}\left\langle\widetilde{\rho}_{k_{2}}^{l_{2}}\left(\mathbf{q}_{2}\right)\right\rangle_{\mathrm{jell}} \\
= & \mathrm{i}^{2} \sum_{m_{1}, m_{2}} \sum_{\mathbf{p}_{1}, \alpha_{1}} \sum_{\mathbf{p}_{2}, \alpha_{2}} \sum_{\mathbf{p}_{3}, \alpha_{3}} \sum_{\mathbf{p}_{4}, \alpha_{4}}\left\langle\mathbf{p}_{1}, \alpha_{1}\left|\mathrm{e}^{\mathrm{i} \mathbf{q}_{1} \mathbf{r}_{||}+\mathrm{i} k_{1} z}\right| l_{1}, m_{1}\right\rangle\left\langle l_{1}, m_{1} \mid \mathbf{p}_{2}, \alpha_{2}\right\rangle \\
& \times\left\langle\mathbf{p}_{3}, \alpha_{3}\left|\mathrm{e}^{\mathrm{i} \mathbf{q}_{2} \mathbf{r}_{||}+\mathrm{i} k_{2} z}\right| l_{2}, m_{2}\right\rangle\left\langle l_{2}, m_{2} \mid \mathbf{p}_{4}, \alpha_{4}\right\rangle \\
& \times\left[\left\langle a_{\alpha_{1}}^{\dagger}\left(\mathbf{p}_{1}\right) a_{\alpha_{3}}^{\dagger}\left(\mathbf{p}_{3}\right) a_{\alpha_{4}}\left(\mathbf{p}_{4}\right) a_{\alpha_{2}}\left(\mathbf{p}_{2}\right)\right\rangle_{\mathrm{jell}}-\left\langle a_{\alpha_{1}}^{\dagger}\left(\mathbf{p}_{1}\right) a_{\alpha_{4}}\left(\mathbf{p}_{4}\right)\right\rangle_{\mathrm{jell}} \delta_{\mathbf{p}_{2}, \mathbf{p}_{3}} \delta_{\alpha_{2}, \alpha_{3}}\right] \\
& -\mathfrak{M}_{k_{1}}^{l_{1}}\left(\mathbf{q}_{1}\right) \mathfrak{M}_{k_{2}}^{l_{2}}\left(\mathbf{q}_{2}\right) \\
= & \mathrm{i}^{2} \frac{N(N-1)}{V^{2}} \sum_{m_{1}, m_{2}} \int \mathrm{d} \mathbf{r}_{1} \int \mathrm{d} \mathbf{r}_{1}^{\prime} \int \mathrm{d} \mathbf{r}_{2} \int \mathrm{d} \mathbf{r}_{2}^{\prime} \mathrm{e}^{\mathrm{i} \mathbf{q}_{1} \mathbf{r}_{|| 1}+\mathrm{i} k_{1} z_{1}+\mathrm{i} \mathbf{q}_{2} \mathbf{r}_{|| 2}+\mathrm{i} k_{2} z_{2}} \\
& \times Y_{l_{1}, m_{1}}\left(\theta_{1}, \phi_{1}\right) Y_{l_{2}, m_{2}}\left(\theta_{2}, \phi_{2}\right) \mathfrak{F}_{2}\left(\mathbf{r}_{1}, \mathbf{r}_{2} \mid \mathbf{r}_{1}^{\prime}, \mathbf{r}_{2}^{\prime}\right) Y_{l_{2}, m_{2}}^{*}\left(\theta_{1}^{\prime}, \phi_{1}^{\prime}\right) Y_{l_{1}, m_{1}}^{*}\left(\theta_{2}^{\prime}, \phi_{2}^{\prime}\right) \\
& -\mathrm{i}^{2} \frac{N}{V} \sum_{m_{1}, m_{2}} \int \mathrm{d} \mathbf{r}_{1} \int \mathrm{d} \mathbf{r}_{1}^{\prime} \mathrm{e}^{\mathrm{i} \mathbf{q}_{1} \mathbf{r}_{|| 1}+\mathrm{i} k_{1} z_{1}} Y_{l_{1}, m_{1}}\left(\theta_{1}, \phi_{1}\right) \\
& -\mathfrak{M}_{k_{1}}^{l_{1}}\left(\mathbf{q}_{1}\right) \mathfrak{M}_{k_{2}}^{l_{2}}\left(\mathbf{q}_{2}\right) .
\end{aligned}
$$

In a similar way, it is possible to present the $n$th order irreducible correlation functions of electrons through the one-, two-, $\ldots, n$-particle density matrices.

Thus, the calculation of the partial function is reduced to the calculation of the many-particle density matrices. Further it will be shown that in case of a local pseudo-potential it is necessary to know only diagonal elements of the many-particle density matrices. They are many-particle distribution functions of electrons which were already examined by us in [22]. The calculation of the many-particle density matrices will be made in future. 


\section{Partial case: local pseudo-potential}

In partial case of the local pseudo-potential $w\left(\left|\mathbf{r}_{i}-\mathbf{R}_{j}\right|\right)$ the function $f_{l}\left(\left|\mathbf{r}_{i}-\mathbf{R}_{j}\right|\right)$ does not depend on an orbital quantum number $l$ :

$$
f_{l}\left(\left|\mathbf{r}_{i}-\mathbf{R}_{j}\right|\right) \equiv f\left(\left|\mathbf{r}_{i}-\mathbf{R}_{j}\right|\right)
$$

and

$$
w\left(\left|\mathbf{r}_{i}-\mathbf{R}_{j}\right|\right)=-\frac{\mathcal{Z} e^{2}}{\left|\mathbf{r}_{i}-\mathbf{R}_{j}\right|}+f\left(\left|\mathbf{r}_{i}-\mathbf{R}_{j}\right|\right)
$$

Then we get

$$
\begin{aligned}
\langle\mathcal{S}(\beta)\rangle_{\text {jell }}= & \exp \left[\sum_{n=1}^{\infty}\left(\frac{\beta N_{\text {ion }}}{S L}\right)^{n} \frac{\mathrm{i}^{n}}{n !} \sum_{\mathbf{q}_{1}, \ldots, \mathbf{q}_{n}} \sum_{k_{1}, \ldots, k_{n}} \mathrm{~S}_{k_{1}}\left(\mathbf{q}_{1}\right) \ldots \mathrm{S}_{k_{n}}\left(\mathbf{q}_{n}\right)\right. \\
& \left.\times \delta w_{k_{1}}\left(\mathbf{q}_{1}\right) \ldots \delta w_{k_{n}}\left(\mathbf{q}_{n}\right) \mathfrak{M}_{k_{1}, \ldots, k_{n}}\left(\mathbf{q}_{1}, \ldots, \mathbf{q}_{n}\right)\right]
\end{aligned}
$$

The result (40) can be written down as

$$
\langle\mathcal{S}(\beta)\rangle_{\text {jell }}=\exp \left[-\beta\left(\delta E^{(1)}+\delta E^{(2)}+\ldots\right)\right]
$$

where $\delta E^{(i)}$ is the energy of the electronic subsystem in the field of the ions minus that in the field of semi-infinite uniform charge background in the $i$ th order approximation with respect to the "difference potential" $\delta w$.

Let us consider this energy in the first order approximation with respect to $\delta w$.

$$
\delta E^{(1)}=-\mathrm{i} \frac{N_{\text {ion }}}{S L} \sum_{\mathbf{q}_{1}} \sum_{k_{1}} \mathrm{~S}_{k_{1}}\left(\mathbf{q}_{1}\right) \delta w_{k_{1}}\left(\mathbf{q}_{1}\right) \mathfrak{M}_{k_{1}}\left(\mathbf{q}_{1}\right) .
$$

In this case

$$
\mathfrak{M}_{k_{1}}\left(\mathbf{q}_{1}\right)=\sum_{l_{1}} \mathfrak{M}_{k_{1}}^{l_{1}}\left(\mathbf{q}_{1}\right)=\mathrm{i} \frac{N}{V} \int \mathrm{d} \mathbf{r}_{1} \mathrm{e}^{\mathrm{i} \mathbf{q}_{1} \mathbf{r}_{|| 1}+\mathrm{i} k_{1} z_{1}} \mathfrak{F}_{1}\left(\mathbf{r}_{1} \mid \mathbf{r}_{1}\right)
$$

$\mathfrak{F}_{1}\left(\mathbf{r}_{1} \mid \mathbf{r}_{1}\right)$ is the one-particle distribution function $F_{1}\left(\mathbf{r}_{1}\right)$ of electrons in semi-infinite jellium [22]. Since semi-infinite jellium is uniform in a plane parallel to the surface, then $F_{1}\left(\mathbf{r}_{1}\right) \equiv F_{1}\left(z_{1}\right)$ and we can rewrite $(42)$ as

$$
\mathfrak{M}_{k_{1}}\left(\mathbf{q}_{1}\right)=\mathrm{i} \frac{N}{V} \int \mathrm{d} \mathbf{r}_{1} \mathrm{e}^{\mathrm{i} \mathbf{q}_{1} \mathbf{r}_{|| 1}+\mathrm{i} k_{1} z_{1}} F_{1}\left(\mathbf{r}_{1}\right)=\mathrm{i} \frac{N}{V} S \delta_{\mathbf{q}_{1}, 0} \int \mathrm{d} z_{1} \mathrm{e}^{\mathrm{i} k_{1} z_{1}} F_{1}\left(z_{1}\right) .
$$

Substituting (43) in (41), we get

$$
\delta E^{(1)}=\frac{N}{V} \sum_{j=1}^{N_{\text {ion }}} \int \mathrm{d} z_{1} \delta w\left(\mathbf{q}=0 \mid z_{1}-Z_{j}\right) F_{1}\left(z_{1}\right)
$$

As

$$
\delta w\left(\mathbf{q}=0 \mid z_{1}-Z_{j}\right)=\int \mathrm{d} \mathbf{r}_{\|} \delta w\left(\sqrt{r_{\|}^{2}+\left(z_{1}-Z_{j}\right)^{2}}\right)=S\langle\delta w\rangle_{\text {plane }}\left(z_{1}-Z_{j}\right),
$$

is held where

$$
\langle\delta w\rangle_{\text {plane }}\left(Z_{j}-z\right)=\frac{1}{S} \int \mathrm{d} \mathbf{r}_{\|} \delta w\left(\sqrt{r_{\|}^{2}+\left(Z_{j}-z\right)^{2}}\right)
$$


is $\delta w$ averaged along the plane parallel to the surface, we can get

$$
\delta E^{(1)}=S \sum_{j=1}^{N_{\text {ion }}} \int \mathrm{d} z_{1}\langle\delta w\rangle_{\text {plane }}\left(z_{1}-Z_{j}\right) n\left(z_{1}\right)
$$

where

$$
n\left(z_{1}\right)=\frac{N}{V} F_{1}\left(z_{1}\right)
$$

is the electron density function of interacting electron gas. The expression for the energy in the first order (46) coincides with that used in works [2-7].

Let us consider this energy in the second order approximation with respect to $\delta w$

$$
\delta E^{(2)}=\beta \frac{N_{\text {ion }}^{2}}{(S L)^{2}} \sum_{\mathbf{q}_{1}, \mathbf{q}_{2}} \sum_{k_{1}, k_{2}} \mathrm{~S}_{k_{1}}\left(\mathbf{q}_{1}\right) \delta w_{k_{1}}\left(\mathbf{q}_{1}\right) \mathrm{S}_{k_{2}}\left(\mathbf{q}_{2}\right) \delta w_{k_{2}}\left(\mathbf{q}_{2}\right) \mathfrak{M}_{k_{1}, k_{2}}\left(\mathbf{q}_{1}, \mathbf{q}_{2}\right) .
$$

In this case

$$
\begin{aligned}
\mathfrak{M}_{k_{1}, k_{2}}\left(\mathbf{q}_{1}, \mathbf{q}_{2}\right)= & \sum_{l_{1}, l_{2}} \mathfrak{M}_{k_{1}, k_{2}}^{l_{1}, l_{2}}\left(\mathbf{q}_{1}, \mathbf{q}_{2}\right) \\
= & \mathrm{i}^{2} \frac{N(N-1)}{V^{2}} \int \mathrm{d} \mathbf{r}_{1} \int \mathrm{d} \mathbf{r}_{2} \mathrm{e}^{\mathrm{i} \mathbf{q}_{1} \mathbf{r}_{|| 1}+\mathrm{i} k_{1} z_{1}+\mathrm{i} \mathbf{q}_{2} \mathbf{r}_{|| 2}+\mathrm{i} k_{2} z_{2}} F_{2}\left(\mathbf{r}_{1}, \mathbf{r}_{2}\right) \\
& -\mathrm{i}^{2} \frac{N}{V} S \delta_{\mathbf{q}_{1}+\mathbf{q}_{2}, 0} \int \mathrm{d} z_{1} \mathrm{e}^{\mathrm{i}\left(k_{1}+k_{2}\right) z_{1}} F_{1}\left(z_{1}\right) \\
& -\mathrm{i}^{2} \frac{N^{2}}{V^{2}} S^{2} \delta_{\mathbf{q}_{1}, 0} \delta_{\mathbf{q}_{2}, 0} \int \mathrm{d} z_{1} \mathrm{e}^{\mathrm{i} k_{1} z_{1}} F_{1}\left(z_{1}\right) \int \mathrm{d} z_{2} \mathrm{e}^{\mathrm{i} k_{2} z_{2}} F_{1}\left(z_{2}\right),
\end{aligned}
$$

where $F_{2}\left(\mathbf{r}_{1}, \mathbf{r}_{2}\right) \equiv \mathfrak{F}_{1}\left(\mathbf{r}_{1}, \mathbf{r}_{2} \mid \mathbf{r}_{1}, \mathbf{r}_{2}\right)$ is the two-particle distribution function of electrons in semiinfinite jellium [22]. Substituting (48) in (47), we get

$$
\begin{aligned}
\delta E^{(2)}= & -\beta \frac{N(N-1)}{2 V^{2}} \sum_{j=1}^{N_{\text {ion }}} \sum_{i=1}^{N_{\text {ion }}} \int \mathrm{d} \mathbf{r}_{1} \int \mathrm{d} \mathbf{r}_{2} \delta w\left(\left|\mathbf{r}_{1}-\mathbf{R}_{j}\right|\right) \delta w\left(\left|\mathbf{r}_{2}-\mathbf{R}_{i}\right|\right) F_{2}\left(\mathbf{r}_{1}, \mathbf{r}_{2}\right) \\
& +\beta \frac{N}{2 V} \sum_{j=1}^{N_{\text {ion }}} \sum_{i=1}^{N_{\text {ion }}} \int \mathrm{d} \mathbf{r} \delta w\left(\left|\mathbf{r}-\mathbf{R}_{j}\right|\right) \delta w\left(\left|\mathbf{r}-\mathbf{R}_{i}\right|\right) F_{1}(z) \\
& +\beta \frac{N^{2}}{2 V^{2}} S^{2} \sum_{j=1}^{N_{\text {ion }}} \int \mathrm{d} z_{1}\langle\delta w\rangle_{\text {plane }}\left(z_{1}-Z_{j}\right) F_{1}\left(z_{1}\right) \sum_{i=1}^{N_{\text {ion }}} \int \mathrm{d} z_{2}\langle\delta w\rangle_{\text {plane }}\left(z_{2}-Z_{i}\right) F_{1}\left(z_{2}\right) .
\end{aligned}
$$

As a rule, all calculations of energy are carried out in the first order in the pseudopotential [7]. In contrast, our approach has no basic difficulties in taking into consideration the second order and higher.

\section{Conclusion}

The theory of semi-infinite metal is presented which takes into consideration the discreteness of an ionic subsystem of metal. The semi-infinite model is used as the reference system for building the perturbation theory powers of the "difference potential" (see footnote on page 642). Consideration of non-local pseudo-potential is the main novelty in this theory. In the specific case of a local pseudopotential, this theory coincides with Kaim's theory. It makes possible to consider structurallydepending contributions to the energy of an electronic subsystem owing to indirect interaction of ions through non-uniform electronic subsystem, interactions of ion groups with non-uniform electronic subsystem. This is very important in order to understand the formation of the metal 
static structure near the surface, to make research into interionic interactions and dynamics of ions close to the surface, as well as to understand the effect of surface structure on surface energy, etc.

Generally, the structurally-depending contributions to energy of an electronic subsystem are expressed through $s$-particle electron density matrices. However, in case of a local pseudo-potential, it is necessary to know only their diagonal elements, that is electron distribution functions. Hence non-locality of a pseudo-potential leads to the necessity of considering non-diagonal elements of density matrices.

\section{A. The proof of an equality}

$$
\left\langle\tilde{\rho}_{k_{1}}^{l_{1}}\left(\mathbf{q}_{1}\right) \ldots \tilde{\rho}_{k_{n}}^{l_{n}}\left(\mathbf{q}_{n}\right)\right\rangle_{\text {jell }}=\left\langle\mathbf{T} \tilde{\rho}_{k_{1}}^{l_{1}}\left(\mathbf{q}_{1} \mid \nu=0\right) \ldots \tilde{\rho}_{k_{n}}^{l_{n}}\left(\mathbf{q}_{n} \mid \nu=0\right)\right\rangle_{\text {jell }}
$$

According to the definition (28)

$$
\begin{aligned}
\left\langle\tilde{\rho}_{k_{1}}^{l_{1}}\left(\mathbf{q}_{1}\right) \ldots \tilde{\rho}_{k_{n}}^{l_{n}}\left(\mathbf{q}_{n}\right)\right\rangle_{\text {jell }}= & \frac{1}{\Xi_{\text {jell }}} \operatorname{Sp}\left(\mathrm{e}^{-\beta \mathcal{H}_{\text {jell }}} \tilde{\rho}_{k_{1}}^{l_{1}}\left(\mathbf{q}_{1}\right) \ldots \tilde{\rho}_{k_{n}}^{l_{n}}\left(\mathbf{q}_{n}\right)\right) \\
= & \frac{1}{\Xi_{\text {jell }}} \frac{(-1)^{n}}{\beta^{n}} \frac{\partial}{\partial \gamma_{k_{1}}^{l_{1}}\left(\mathbf{q}_{1}\right)} \cdots \frac{\partial}{\partial \gamma_{k_{n}}^{l_{n}}\left(\mathbf{q}_{n}\right)} \\
& \times \operatorname{Sp} \exp \left(-\beta \mathcal{H}_{\text {jell }}-\beta \sum_{\mathbf{q}, k, l} \gamma_{k}^{l}(\mathbf{q}) \tilde{\rho}_{k}^{l}(\mathbf{q})\right) \mid\left\{\begin{array}{c}
\gamma_{k_{1}}^{l_{1}}\left(\mathbf{q}_{1}\right)=0 \\
\ldots \\
\gamma_{k_{n}}^{l_{n}}\left(\mathbf{q}_{n}\right)=0
\end{array}\right.
\end{aligned}
$$

Transferring to the interaction representation we get

$$
\begin{aligned}
\left\langle\tilde{\rho}_{k_{1}}^{l_{1}}\left(\mathbf{q}_{1}\right) \ldots \tilde{\rho}_{k_{n}}^{l_{n}}\left(\mathbf{q}_{n}\right)\right\rangle_{\text {jell }}= & \frac{1}{\Xi_{\text {jell }}} \frac{(-1)^{n}}{\beta^{n}} \frac{\partial}{\partial \gamma_{k_{1}}^{l_{1}}\left(\mathbf{q}_{1}\right)} \cdots \frac{\partial}{\partial \gamma_{k_{n}}^{l_{n}}\left(\mathbf{q}_{n}\right)} \\
& \times \operatorname{Sp}\left(\mathrm{e}^{-\beta \mathcal{H}_{\text {jell }}} \mathrm{T} \exp \left[-\int_{0}^{\beta} \mathrm{d} \beta^{\prime} \sum_{\mathbf{q}, k, l} \gamma_{k}^{l}(\mathbf{q}) \tilde{\rho}_{k}^{l}\left(\mathbf{q} \mid \beta^{\prime}\right)\right]\right) \mid\left\{\begin{array}{c}
\gamma_{k_{1}}^{l_{1}}\left(\mathbf{q}_{1}\right)=0 \\
\ldots \\
\gamma_{k_{n}}^{l_{n}}\left(\mathbf{q}_{n}\right)=0
\end{array}\right. \\
= & \frac{1}{\Xi_{\text {jell }}} \frac{(-1)^{n}}{\beta^{n}} \frac{\partial}{\partial \gamma_{k_{1}}^{l_{1}}\left(\mathbf{q}_{1}\right)} \cdots \frac{\partial}{\partial \gamma_{k_{n}}^{l_{n}}\left(\mathbf{q}_{n}\right)} \\
& \times \operatorname{Sp}\left(\mathrm{e}^{-\beta \mathcal{H}_{\text {jell }}} \mathrm{T} \exp \left[-\beta \sum_{\mathbf{q}, k, l} \gamma_{k}^{l}(\mathbf{q}) \tilde{\rho}_{k}^{l}(\mathbf{q} \mid \nu=0)\right]\right) \mid\left\{\begin{array}{c}
\gamma_{k_{1}}^{l_{1}}\left(\mathbf{q}_{1}\right)=0 \\
\ldots \\
\gamma_{k_{n}}^{l_{n}}\left(\mathbf{q}_{n}\right)=0
\end{array}\right. \\
= & \left\langle\mathrm{T} \tilde{\rho}_{k_{1}}^{l_{1}}\left(\mathbf{q}_{1} \mid \nu=0\right) \ldots \tilde{\rho}_{k_{n}}^{l_{n}}\left(\mathbf{q}_{n} \mid \nu=0\right)\right\rangle_{\text {jell }} .
\end{aligned}
$$

Thus, the statement

$$
\left\langle\tilde{\rho}_{k_{1}}^{l_{1}}\left(\mathbf{q}_{1}\right) \ldots \tilde{\rho}_{k_{n}}^{l_{n}}\left(\mathbf{q}_{n}\right)\right\rangle_{\text {jell }}=\left\langle\mathrm{T} \tilde{\rho}_{k_{1}}^{l_{1}}\left(\mathbf{q}_{1} \mid \nu=0\right) \ldots \tilde{\rho}_{k_{n}}^{l_{n}}\left(\mathbf{q}_{n} \mid \nu=0\right)\right\rangle_{\text {jell }}
$$

is proved. 


\title{
References
}

1. Partenskij M.B. Uspekhi Fiz. Nauk, 1979, 128, 69 (in Russian).

2. Lang N. D., Kohn W., Phys. Rev. B, 1970, 1, No. 12, 4555-4567.

3. Perdew J.P., Monnier R., Phys. Rev. Lett., 1976, 37, No. 19, 1286.

4. Monnier R., Perdew J.P., Phys. Rev. B, 1978, 17, No. 6, 2595.

5. Sahni V., Perdew J.P., Gruenebaum J., Phys. Rev. B, 1981, 23, No. 12, 6512.

6. Kobeleva R.M., Gelchynskij B.R., Uhov V.F., Fiz. Metallov i Metalloved., 1978, 45, No. 1, 25 (in Russian).

7. Fiolhais C., Henriques C., Sarría I, Pitarke J.M., Prog. Surf. Sci. 2001, 67, 285.

8. Rose J.H., Dobson J.F., Solid State Comm., 1981, 37, No. 2, 91-96.

9. Dobson J.F., Rose J.H., J. Phys. C, 1982, 15, 7429-7456.

10. Barnett R.N., Barrera R.G., Cleveland C.L., Landman U., Phys. Rev. B, 1983, 28, No. 4, 1667.

11. Eguiluz A.G., Phys. Rev. B, 1987, 35, No. 11, 5473.

12. Kaim S.D., Ukr. Fiz. Zhurn., 1981, 26, No. 8, 1351 (in Russian).

13. Kiejna A., Phys. Rev. B, 1993, 47, No. 12, 7361.

14. Kiejna A., Ziesche P., Kaschner R., Phys. Rev. B, 1993, 48, No. 7, 4811.

15. Fiolhais C., Perdew J.P., Phys. Rev. B, 1992, 45, No. 11, 6204.

16. Browman E.G., Kagan Yu.M., Holas A., Fiz. Tv. Tela, 1970, 12, No. 4, 1001 (in Russian).

17. Gurskij Z.A., Krasko G.L., Fiz. Tv. Tela, 1969, 11, No. 10, 2717 (in Russian).

18. Gurskij Z.A., Krasko G.L., Doklady Akad. Nauk SSSR, 1971, 197, No. 4, 810 (in Russian).

19. Gurskij Z.A., Fiz. Metallov i Metalloved., 1973, 36, No. 2, 265 (in Russian).

20. Kostrobij P.P., Markovych B.M., Condens. Matter Phys., 2003, 6, No. 2(34), 347-362.

21. Kostrobij P.P., Markovych B.M., J. Phys. Stud., 2003, 7, No. 2, 195-206 (in Ukrainian).

22. Kostrobij P.P., Markovych B.M., J. Phys. Stud., 2003, 7, No. 3, 298-312 (in Ukrainian).

23. Kostrobij P.P., Markovych B.M., Condens. Matter Phys., 2006, 9, No. 4(48), 747-756; Kostrobij P.P., Markovych B.M. Preprint of the Institute for Condensed Matter Physics, ICMP-06-05U, Lviv, 2006 , $18 \mathrm{p}$ (in Ukrainian).

24. Yakibchuk P.M., Condens. Matter Phys., 1997, 10, 179-187.

25. Bogoljubov N.N. The selected works in three volumes. Naukova dumka, Kiev, 1970 (in Russian).

\section{Напівобмежений метал: підхід на основі моделі напівобмеженого "желе"}

\author{
П.П.Костробій, Б.М.Маркович \\ Національний університет “Львівська політехніка”, вул. С. Бандери, 12, Львів 79013 \\ Отримано 27 лютого 2008 р.
}

Представлено енергію електронної підсистеми напівобмеженого металу у вигляді ряду за степенями псевдопотенціалу. Показано, що в загальному випадку для розрахунку цієї енергії необхідні електронні багаточастинкові матриці густини, тоді як у частковому випадку локального псевдопотенціалу лише її діагональні елементи (функції розподілу електронів). У першому порядку теорії збурень у випадку локального псевдопотенціалу наш результат для енергії співпадає із широко застосовуваними у теорії функціоналу густини.

Ключові слова: велика статистична сума, псевдопотенціал, багаточастинкова матриця густини

PACS: $71.45 . G m, 71.10 .-w, 73.20 .-r$ 
